\title{
VIABILITY OF VASCULARIZED BONE GRAFT FROM THE ILIAC CREST USING THE ILIAC BRANCH OF THE ILIOLUMBAR ARTERY: EXPERIMENTAL STUDY ON RATS
}

Fabian Maccarini Peruchi ${ }^{1}$, Alessandra Deise Sebben², Martina Lichtenfels ${ }^{3}$, Marcos Ricardo de Oliveira Jaeger ${ }^{4}$, Jefferson Braga Silva ${ }^{5}$

\section{ABSTRACT}

Objective: Through an experimental model, our aim was to create inferences about the viability of vascularized bone grafts from the iliac crest in rats and investigate their histological features. Methods: Twenty-one rats were used, divided into two groups: the first consisted of animals that were subjected to the technique of vascularized bone graft pedicled onto the iliac branch of the iliolumbar artery; the second (control group) underwent the same procedure as performed on the first group, with the addition of ligation of the vascular pedicle. The viability of the bone grafts was observed for three weeks, by means of direct observation of the graft, histology and immunohistochemistry. Results: All the vascularized grafts evaluated in the first week showed viability according to direct observation, histology and immunohistochemistry. However, in the second and third weeks, direct observation showed that $75 \%$ of the grafts were unviable, while histological analysis and immunohistochemistry showed that $50 \%$ were unviable. Conclusions: Some grafts that are designed to be vascularized became unviable and began to behave like non-vascularized grafts under direct observation and histology. Despite the possibility of failure, use of vascularized bone grafts should be encouraged, because descriptive histology shows greater cell density in the medullary bone portion, and osteocytes that function better regarding deposition of bone matrix, with preservation of the intraosseous vascular network.

Keywords - Bone Transplantation; Bone Matrix; Histology; Immunohistochemistry; Models, Animal

\section{INTRODUCTION}

Bone grafts continue to be used frequently for resolving clinical situations that involve loss of bone substance. They are very widely applicable in reconstructive surgery, especially within orthopedics, for repairing bone tissue. Such repairs might be consequent to trauma, pseudarthrosis, correction of deformities, tumor resection or stimulation of osteogenesis ${ }^{(1-4)}$.

The viability of the bone cells transferred with the graft is one of the determining factors for the mechanical properties of the graft, such as the resistance to external forces, porosity and bone density, and also physiological properties such as the capacity for osteogenesis and osteoinduction. For this reason, factors that contribute towards cell survival should be respected, in order to obtain superior-quality grafts, thereby leading to better prognoses in interventions that aim to reconstruct skeletal defects.

There is an inherent doubt regarding the surgical procedure when vascularized bone grafts are used: will the bone graft remain viable through the vascular pedicle over the course of time? Certain events may obstruct the pedicle, such as external compression, edema, postoperative immobilization, thrombosis due to venous stasis or pedicle injuries due to manipulation. If the pedicle becomes obstructed, the

1 - MSc in Medicine and Health Sciences, PUCRS, Porto Alegre, RS, Brazil.

2 - Biologist and Doctoral Student in Medicine and Health Sciences, PUCRS, Porto Alegre, RS, Brazil

3 - Biologist and Master's Student in Medicine and Health Sciences, PUCRS, Porto Alegre, RS, Brazil.

$4-\mathrm{PhD}$ in Medicine and Health Sciences, PUCRS, Porto Alegre, RS, Brazil.

5 - Titular Professor of the Department of Surgery, School of Medicine, PUCRS, Porto Alegre, RS, Brazil.

Work performed at the Pontificate Catholic University of Rio Grande do Sul (PUCRS), Porto Alegre, RS.

Correspondence: Av. Ipiranga 6690, conj. 216, Jardim Botânico, 90610-000 Porto Alegre, RS, Brazil. E-mail: jeffmao@terra.com.br

Work received for publication: July 7, 2011; accepted for publication: August 4, 2011.

The authors declare that there was no conflict of interest in conducting this work 
vascularized bone graft will be transformed into a non-vascularized graft.

Through an experimental model in rats, our aim here was to make inferences regarding the viability of a vascularized bone graft from the iliac crest, sustained by a vascular pedicle, and to investigate its histological characteristics.

\section{METHODS}

\section{Animals}

Twenty-one male Wistar rats (Rattus norvegicus) were used. They were from a research-dedicated stock maintained at the Medical Skills and Surgical Research Laboratory of PUCRS, under appropriate environmental conditions for the species, with controlled temperature, humidity, ventilation, light, noise, odor and social interaction. They were kept in individual cages, receiving water and food of quality and quantity that were appropriate for the species. The study protocol had previously been approved by the Ethics Committee for Animal Use of PUCRS.

The animals were divided into two comparison groups: Group 1: vascularized bone graft from the iliac crest $(\mathrm{n}=12)$.

Group 2 (control): non-vascularized bone graft from the iliac crest $(n=9)$.

\section{Surgical procedure}

Anesthesia was administered intramuscularly, consisting of a solution of $0.2 \mathrm{ml}$ of chlorpromazine and $0.8 \mathrm{ml}$ of ketamine, at a dose of $3 \mathrm{ml} / \mathrm{kg}$. Maintenance doses of anesthetic were administered over the course of the surgical procedure, as necessary.

The surgical incision was longitudinal: parallel to and separated by $2 \mathrm{~cm}$ from the dorsal median line, starting $1 \mathrm{~cm}$ below the final costal arch and ending at the level of the hips (Figure 1).

In the subfascial layer, the iliac branch of the iliolumbar vein and artery were identified and carefully dissected in the proximal direction, as far as the point where they entered the abdominal cavity. To access the abdominal cavity, the insertion of the abdominal muscles was released at the iliac crest. The vascular pedicle was dissected as far as its origin in the aortic artery and vena cava inferior (Figure 2).

The branch to the iliac bone was then identified, and the artery was ligated distally to it (Figure 3).

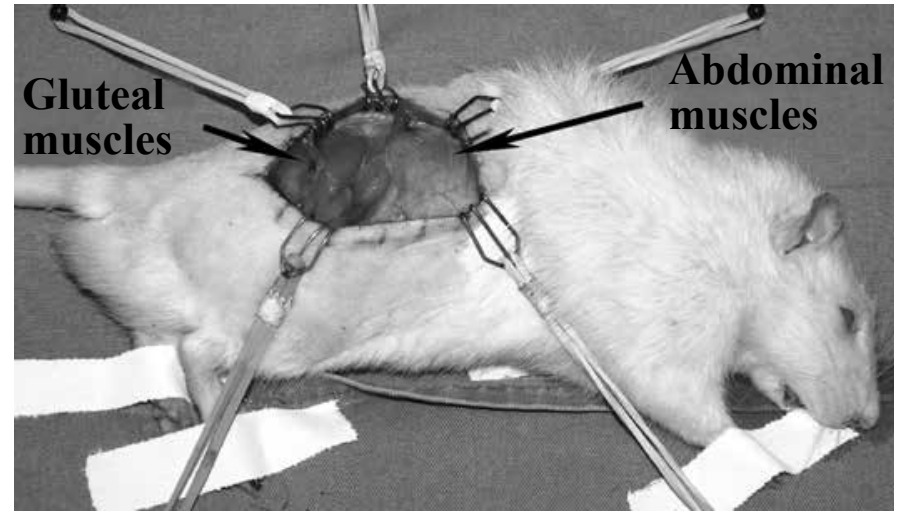

Figure 1 - Surgical access and location of the transition between the gluteal and abdominal musculatures, which were reference points for locating the pedicle.

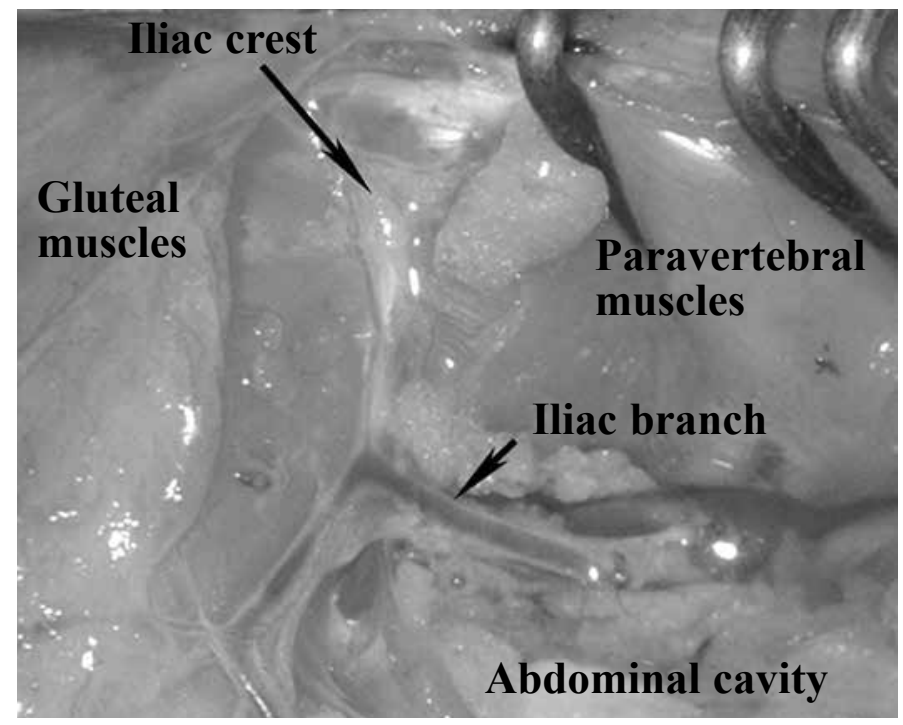

Figure 2 - Laparotomy with dissection of the iliac branch of the iliolumbar artery, towards its origin in the abdominal aorta.

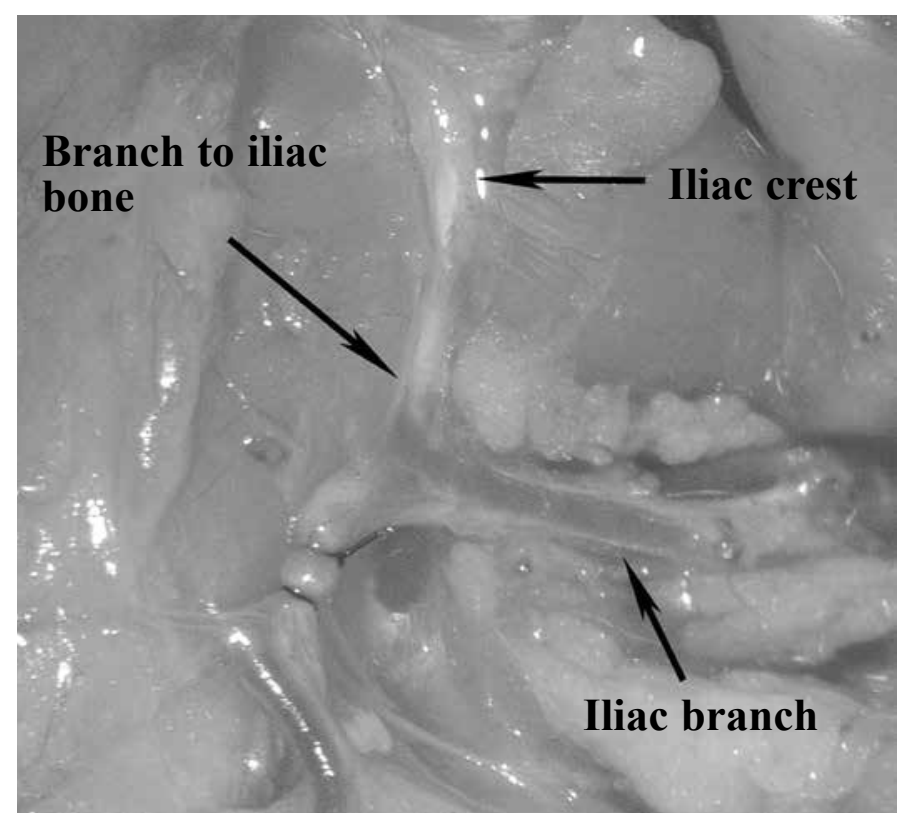

Figure 3 - Ligation of the iliac branch distally to the emergence of the vessel to the iliac bone. 
The thoracolumbar fascia was incised at the lateral margin of the spine and the medial portion of the iliac bone was exposed. Parallel to the upper margin of the iliac bone and $1 \mathrm{~cm}$ from it, the gluteus maximus muscle was incised in the external face of the iliac and the iliac muscle was incised to expose the internal face of the iliac bone (Figure 4).

Bicortical osteotomy separated the upper third of the iliac bone from the lower two thirds, which were jointed to the sacrum and hips. The soft tissues that were inserted into the upper half were released, thus making it possible to produce a bone graft vascularized by the iliac branch of the iliolumbar vein and artery (Figure 5).

The bone graft was wrapped in a silicone lamina, thus creating a mechanical barrier that made inva-

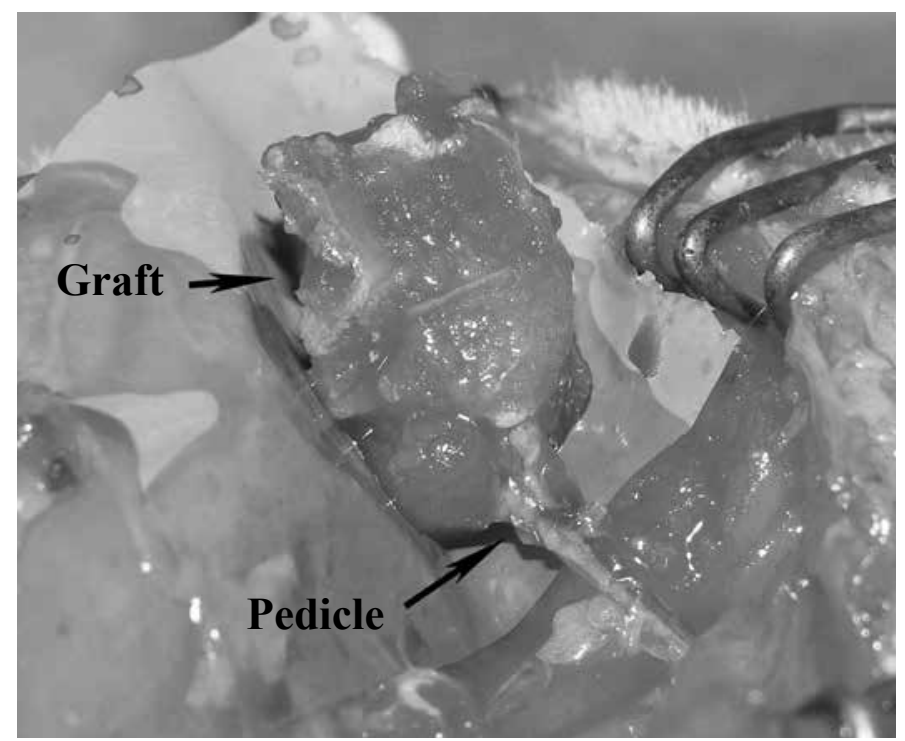

Figure 4 - Vascularized bone graft from the iliac bone based on the iliac branch of the iliolumbar artery.

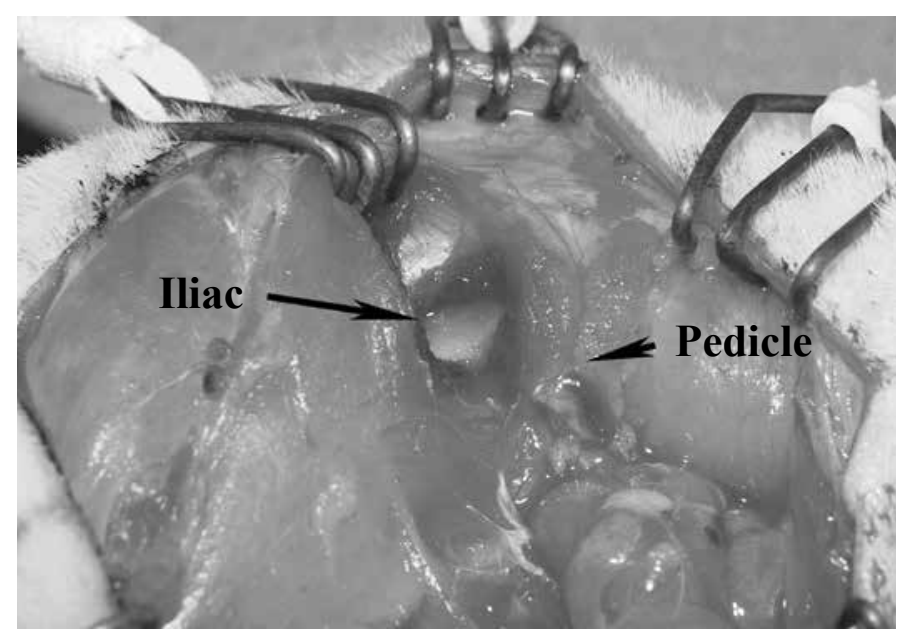

Figure 5 - Exposure of the external face of the iliac bone after incision and separation of the gluteal muscle. sion difficult and diminished the influence of neighboring tissues on bone graft viability, and making the graft dependent on its vascular pedicle for viability to be maintained.

Group 2 was used as a control group. In this, the same procedure as described for group was performed, with the modification that the vascular pedicle was ligated and sectioned.

The graft, wrapped in the silicone lamina, was placed inside the abdominal cavity with the aim of minimizing the chances of extrusion to the external environment.

Among the 21 animals, seven were sacrificed one week after the surgical procedure; seven, two weeks afterwards; and seven, three weeks afterwards. Among the seven animals sacrificed each week, four were in group 1 and the other three were in group 2 .

\section{Histology}

The grafts were fixed in $10 \%$ buffered formalin for 24 hours, and then were washed with water and kept in water for 12 hours and then decalcified for 24 hours, using a solution of $22 \%$ formic acid and $10 \%$ sodium citrate. After this procedure, the specimen was included in paraffin in accordance with the processing for conventional tissues, and histological sections of $3 \mu \mathrm{m}$ were cut and then stained with hematoxylin-eosin (HE).

\section{Immunohistochemistry}

The same specimens that had been fixed and decalcified for histological analysis were used to prepare slides for immunohistochemical analysis. The anti-CD34 endothelial marker was used to assess the intraosseous vascularization.

\section{STATISTICAL ANALYSIS}

The variables were described in terms of absolute and relative frequencies.

To evaluate the concordance between the methods, the kappa concordance coefficient was applied. To estimate the magnitude of the kappa coefficient, the $95 \%$ confidence interval was calculated.

The significance level adopted was $5 \%$, and the analyses were performed using the SPSS software (Statistical Package for the Social Sciences), version 13.0, and the PEPI software (Programs for Epidemiologists), version 4.0. 


\section{RESULTS}

\section{Direct observation}

Differences in macroscopic characteristics between the vascularized and non-vascularized grafts were evident. In the vascularized grafts, a piece of bone of reddish tone was found, with abundant bone marrow that was bleeding. On manipulation, it presented plasticity similar to that of the bone encountered at the time when the initial surgical procedure had been performed, albeit with loss of resistance. The nonvascularized bone grafts presented a yellowish-white coloring, with sparse bone marrow, and they were less plastic and more friable, without any evidence of bleeding, and a dried-out appearance.

In all the rats whose procedure was aimed towards obtaining a non-vascularized graft, bone portions with the above-described characteristics were observed via ectoscopy. However, among the rats whose procedure was aimed towards integrity of graft vascularization, all those sacrificed in the first week were characterized via ectoscopy as vascularized. Only one was classified as vascularized among those of the second and third weeks. Thus, vascularization was seen to be absent in $75 \%$ (three out of four) of the grafts that had initially been vascularized, in the second and third weeks.

\section{Histology}

From observations on the slides stained with hematoxylin-eosin (Figure 6), the bones could be classified as vascularized or non-vascularized, according to the structures viewed. In the vascularized grafts, well-structured trabeculae were found in the bone marrow region, and large quantities of osteocytes with abundant deposition of osteoid matrix surrounding it, and with a high density of cellular elements in the bone marrow region. On the other hand, situations in which a slide with smaller quantities of osteocytes were found, accompanied by smaller quantities of bone matrix deposited around them, and sparse bone marrow, were characterized as a non-vascularized bone pattern. All the slides from group 2 were classified as non-vascularized. The slides from the histological specimens from group 1 in the first week were classified as vascularized. However, only $50 \%$ (two out of four) of the slides obtained afterwards, in the second and third weeks, were classified as vascularized.

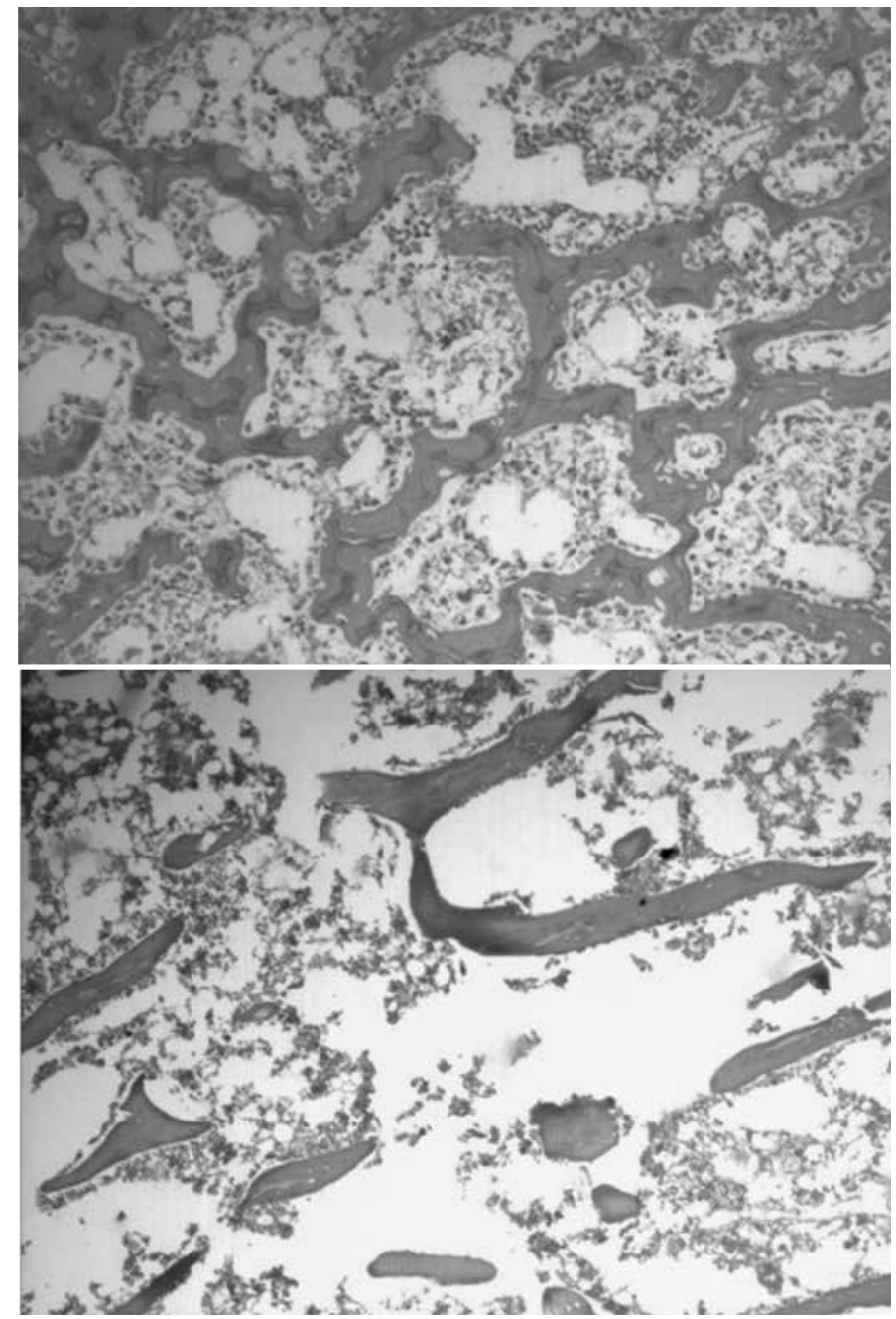

Figure 6 - Photomicrograph of histological section through a bone graft with the characteristics of viability (left), in which the bone marrow region presents abundant cellular elements and osteogenic matrix; and section showing absence of viability (right), in which there are signs of bone reabsorption and diminished cell density in the bone marrow $(\mathrm{HE}, 100 \mathrm{x})$.

The slides stained with the immunohistochemical marker anti-CD 34 for endothelial tissue also showed differences in the histological descriptive analysis. In assessing the slides that were classified as presenting the vascularized bone pattern, a large quantity of structures marked with brown staining (staining due to DAB) were found, with the presence of a lumen containing erythrocytes, thus characterizing blood vessels. The slides with non-vascularized bone showed absence of the above-described structures. None of the slides from group 2 showed structures corresponding to blood vessels. Among the slides from vascularized grafts, all of those from the first week demonstrated signs of vascularization, while only $50 \%$ of the slides from the second and third weeks had blood vessels. 


\section{Correlation between direct observation and histology}

There was statistically significant concordance between all the methods $(\mathrm{p}<0.001)$, as presented in Table 1. It was observed that the kappa coefficient presented good concordance between all the methods $(\geq 0.79)$, with complete concordance between the hematoxylin-eosin and CD34 staining $(\mathrm{k}=1.00)$. Only two of the 21 evaluations $(9.5 \%)$ were discordant between direct observation and histology using hematoxylin-eosin and CD34 staining. In these two cases, direct observation demonstrated devascularization, while the other methods presented vascularization.

Table 1 - Assessment of the concordance between direct observation, hematoxylin-eosin (HE) and CD34.

\begin{tabular}{c|c|c|c}
\hline \multirow{2}{*}{ Comparison } & Concordance & Kappa (95\% Cl) & p \\
\cline { 2 - 3 } & $\mathbf{n}(\%)$ & $\begin{array}{c}0.79 \\
(0.51-1.00)\end{array}$ & $<0.001$ \\
\hline $\begin{array}{c}\text { Direct observation } \\
\text { vs, HE }\end{array}$ & $19 / 21(90.5)$ & $\begin{array}{c}0.79 \\
(0.51-1.00)\end{array}$ & $<0.001$ \\
\hline $\begin{array}{c}\text { Direct observation } \\
\text { vs, CD 34 }\end{array}$ & $19 / 21(90.5)$ & $\begin{array}{c}1.00 \\
(0.68-1.00)\end{array}$ & $<0.001$ \\
\hline HE vs. CD 34 & $21 / 21(100.0)$ & & \\
\hline
\end{tabular}

\section{DISCUSSION}

Bone grafts are fundamental for reconstruction of skeletal defects. The success of the treatment may be influenced by the type of graft used, because of the differences in properties between vascularized and non-vascularized bone grafts.

The properties of vascularized bone grafts make it possible to achieve survival of a greater proportion of their cellular elements ${ }^{(5)}$, earlier consolidation and integration of the receptor bed ${ }^{(6,7)}$, better maintenance of bone mass ${ }^{(5)}$, greater capacity for hypertrophy ${ }^{(5)}$ and higher resistance to infections in relation to non-vascularized bone grafts ${ }^{(8,9)}$.

Physiologically, vascularized bone grafts present greater capacity for osteogenesis, osteoinduction and osteoconduction, compared with non-vascularized bone grafts, and it becomes clinically imperative to use them in certain situations in which the failure rate with conventional bone grafts would be far too high to justify using them, such as in cases of extensive bone defects, poorly vascularized or even devascularized receptor beds ${ }^{(10,11)}$ and previous procedures using grafts that resulted in failure of the procedure. Nonetheless, if the prognosis improves through using vascularized grafts in the cases with worse prognosis, these may also be used more frequently or even as the first choice in situations that are considered to be less severe.

One frequent question raised regarding vascularized bone grafts is in relation to the viability of the graft with the passage of time: would it be justifiable to choose a technique that prolongs the duration of the surgical procedure or requires referral to professionals with greater skills in performing this procedure? Grafts that initially were proven to be vascularized during the operation may cease to be so for a variety of reasons, for example: venous stasis, vascular microtrauma, coagulation disorders, systemic abnormalities, external compression, edema and inflammatory or infectious processes.

In an experimental study on rats, Linsell et $\mathrm{al}^{(12)}$ reported that $88 \%$ of their vascularized grafts of the femur were viable after one week of follow-up, when they used arteriovenous microanastomosis.

Sempuku et $\mathrm{al}^{(13)}$ detected bone neoformation within two weeks, in a model for vascularized bone grafts in rat tails, such that in the group with non-vascularized grafts, complete necrosis was observed one week after the surgery. Bone neoformation surrounding the necrotic bone was detected five weeks after the surgery, thus suggesting the revascularization and osteogenesis were occurring through invasion by surrounding tissues.

Nasir et $\mathrm{al}^{(14)}$ observed from histological analysis on rats that, after one week, a bone graft from the iliac crest with vascularization from the lateral circumflex femoral artery was viable.

Ozkan et $\mathrm{l}^{(15)}$ used an experimental model in rats, for a vascularized bone graft based on the iliolumbar artery, and found that all the grafts examined were histologically viable. The experimental model of Ozkan et $\mathrm{al}^{(15)}$, which was chosen for the present study, was shown to be feasible and only presented small variations in vessel path, vessel location and number of branches. This constant anatomy made construction of vascularized grafts reliable and simple. Because rats are small-sized animals and consequently, their vessels are small, use of a microsurgical microscope added security in dissecting and ligating the vessels. The main advantages of this model that they cited were the small functional loss for the animal without alterations in gait, the bone fragment of ample 
dimensions $(1 \times 1 \mathrm{~cm})$ that was obtained, the possibility of protecting the bone fragment in the abdominal cavity and the ease of execution.

In this experimental study, it was found during the operation that all the vascularized grafts presented active bleeding, with functional vascular pedicles. At the time of the surgical reintervention to collect material and analyze the results, the animals sacrificed in the first week had grafts that were considered to be vascularized according to both direct observation and histology. However, among the animals of the second week, only one was considered to be vascularized according to direct observation (25\%) and, according to histology, two of them $(50 \%)$. These results were repeated among the animals sacrificed in the third week. There was a correlation between the results from the ectoscopic and histological classifications.

\section{CONCLUSION}

The grafts that were initially vascularized became devascularized at a certain time and started to behave like non-vascularized grafts, under ectoscopic and histological analysis. Further studies would be needed in order to make inferences about the possible causes of the failures of vascularization.

Despite the possibility of failure, use of vascularized bone grafts should be encouraged, since the descriptive histological evaluation showed that these grafts had greater cell density in the bone marrow portion and osteocytes with greater functionality of bone matrix deposition, with a preserved intraosseous vascular network. Thus, bone portions with inherent characteristics that promote greater chances of cure for patients could be used in reconstructive procedures.

\section{REFERENCES}

1. Yajima $H$, Tamai $S$, Mizumoto $S$, Inada $Y$. Vascularized fibular grafts in the treatment of osteomyelitis and infected nonunion. Clin Orthop Relat Res. 1993;(293):256-64.

2. Weiland AJ, Moore JR, Daniel RK. Vascularized bone autografts. Experience with 41 cases. Clin Orthop Relat Res. 1983;(174):87-95.

3. Wood MB, Cooney WP 3rd. Vascularized bone segment transfers for management of chronic osteomyelitis. Orthop Clin North Am. 1984 Jul;15(3):461-72.

4. Wood MB, Bishop AT. Massive bone defects of the upper limb: reconstruction by vascularized bone transfer. Hand Clin. 2007;23(1):49-56.

5. Tamai S. Experimental vascularized bone transplantations. Microsurgery. 1995; 16(4):179-85

6. Haw CS, O'Brien BM, Kurata T. The microsurgical revascularisation of resected segments of tibia in the dog. J Bone Joint Surg Br. 1978;60(2):266-9.

7. Weiland AJ, Phillips TW, Randolph MA. Bone grafts: a radiologic, histologic, and biomechanical model comparing autografts, allografts, and free vascularized bone grafts. Plast Reconstr Surg. 1984;74(3):368-79.

8. Cutting $\mathrm{CB}, \mathrm{McC}$ arthy JG. Comparison of residual osseous mass between vascularized and nonvascularized onlay bone transfers. Plast Reconstr Surg. 1983;72(5):672-5.
9. Antonyshyn O, Colcleugh RG, Anderson C. Growth potential in onlay bone grafts: a comparison of vascularized and free calvarial bone and suture bone grafts. Plast Reconstr Surg. 1987;79(1):12-23.

10. Cooney WP, Linscheid RL, Dobyns JH, Wood MB. Scaphoid nonunion: role of anterior interpositional bone grafts. J Hand Surg Am. 1988;13(5):635-50.

11. Green DP. The effect of avascular necrosis on Russe bone grafting for scaphoid nonunion. J Hand Surg Am. 1985;10(5):597-605.

12. Linsell M, Jablonski P, Howden B, Scott D, Marshall V. The thigh flap: an osteomyocutaneous free-flap model in the rat. Plast Reconstr Surg. 1988;81(2):240-5.

13. Sempuku T, Tamai $S$, Mizumoto $S$, Yajima $H$. Vascularized tail bone grafts in rats. Plast Reconstr Surg. 1993;91(3):502-10.

14. Nasir $S$, Aydin A, Kayikçioğlu A, Sökmensüer $C$, Cobaner A. New experimental composite flap model in rats: gluteus maximus-tensor fascia lata osteomuscle flap. Microsurgery. 2003;23(6):582-8.

15. Ozkan O, Akyürek M, Safak T, Kayikçioğlu A, Güler G, Erk Y. A new flap model in rats: iliac osteomusculocutaneous flap. Ann Plast Surg. 2001;47(2):161-7. 